\title{
Treatment of a Frail Older Patient with Diffuse Large B-Cell Lymphoma on Maintenance Dialysis: Attenuated Immunochemotherapy and Adapted Care Plan
}

\author{
Luciola de Barros Pontes ${ }^{a} \quad$ Juliana Todaro ${ }^{b, e}$ Theodora Karnakis ${ }^{c, f}$ \\ Patricia Weinschenker Bollman ${ }^{\mathrm{b}, \mathrm{e}}$ Bento Fortunato Cardoso Santos ${ }^{\mathrm{d}}$ \\ Rafael Aliosha Kaliks ${ }^{a}$ Auro Del Giglio ${ }^{a, e}$ \\ Departments of ${ }^{\mathrm{a} C l i n i c a l}$ Oncology, ${ }^{\mathrm{b}} \mathrm{Hematology},{ }^{\mathrm{c}}$ Geriatric Oncology and ${ }^{\mathrm{d}} \mathrm{Nephrology,}$ \\ Albert Einstein Hospital, ${ }^{\mathrm{e}}$ Faculdade de Medicina do $A B C$, and ${ }^{\mathrm{f}}$ Sao Paulo Institute of \\ Cancer (ICESP), Sao Paulo, Brazil
}

\section{Key Words}

Lymphoma · Elderly · Comprehensive geriatric assessment · Chemotherapy · Renal failure

\begin{abstract}
Purpose of the Study: Diffuse large B-cell lymphoma (DLBCL) is the most common lymphoid malignancy worldwide. Its incidence increases with age and about $40 \%$ of cases occur in patients over 70 years. Herein, we describe a case of a frail elderly patient with renal insufficiency and DLBCL treated with R-mini-CHOP. Case Report: A 77-year-old man on maintenance hemodialysis started experiencing persistent fatigue. He was diagnosed with a large mass on the left lobe of the lung. Biopsy demonstrated a DLBCL, CD20 positive. The patient was assigned clinical stage IIBX, with a high age-adjusted international prognosis index. A proper geriatric assessment revealed a frail patient. Thus, an adapted chemotherapy regimen was proposed which consisted of R-mini-CHOP every 21 days, with a reduction of $10 \%$ in the doses of doxorubicin and cyclophosphamide. During the treatment, the patient went through regular dialysis sessions, 3 times per week, with an extra session performed 12 $\mathrm{h}$ after each chemotherapy administration. The patient experienced no adverse events or grade 3/4 toxicities. After 6 cycles of R-mini-CHOP, the patient achieved unconfirmed complete remission, and consolidation radiotherapy was performed. At the last follow-up, he was still in unconfirmed complete remission, with a progression-free survival of 11.3 months. Conclusions: $\mathrm{R}-$ mini-CHOP represented a reasonable treatment option for this patient with
\end{abstract}


renal failure. The oncogeriatric approach led to a successful management of this frail patient, highlighting that an adapted plan of care is a key issue to improve the outcomes of elderly cancer patients.

\section{Introduction}

Life expectancy has grown over the last decades, and further increases are projected. By the year 2030, the population older than 75 years will be multiplied by three, and of those over 85 years of age there will be twice as many as today [1]. Considering that ageing is associated with an increment in cancer incidence, a specific approach will be required to deal with these patients.

Diffuse large B-cell lymphoma (DLBCL) is the most common lymphoid malignancy worldwide [2]. Its incidence increases with age and about $40 \%$ of cases occur in patients over 70 years [2]. The prognosis of elderly patients with lymphoma is worse when compared to their younger counterparts, particularly for those with aggressive subtypes, as a consequence of the difficulties encountered during treatment and even before choosing the adequate treatment. As a result of these factors, and sometimes simply because of their age, elderly lymphoma patients are often treated with regimens with low doses of standard chemotherapeutics or less toxic but also less active drugs [3].

Similarly, tolerance to treatment is also affected by physiological changes related to ageing, such as declining renal function. As expected, there is a paucity of data on the management of cancer patients with renal impairment, and the treatment approach to nonHodgkin's lymphoma patients undergoing maintenance hemodialysis (HD) is a challenging concern.

Nevertheless, several trials show that a treatment with curative intent is still possible in elderly patients, and adapted chemotherapy can offer long life expectancy [4-6]. Peyrade et al. [4], in a prospective trial of the Groupe d'Etude des Lymphomes de l'Adulte (GELA), proposed an attenuated regimen of treatment based on the classic chemotherapy regimen $\mathrm{R}$ CHOP, the so-called R-mini-CHOP, as standard treatment for elderly lymphoma patients with good performance status.

There is little data regarding the management of frail elderly patients with DLBCL, and even less is known about the treatment of such patients in HD. Thus, we describe a case of a frail 77-year-old patient with renal insufficiency and DLBCL treated with an attenuated immunochemotherapy after an adapted care plan guided by a comprehensive geriatric assessment (CGA).

\section{Case Report}

A 77-year-old man, on maintenance HD for 4 years, started experiencing persistent fatigue. His physical examination was unremarkable and the imaging workup with a chest tomography revealed a heterogeneous $8.3 \times 9.8 \mathrm{~cm}^{2}$ mass on the left upper lobe of the lung, as well as mediastinal lymph nodes. A biopsy was done and pathology demonstrated DLBCL, CD20 positive, Ki67 95\%. After staging with an FDG PET/CT (positron-emission tomography with 2-[fluorine-18]-fluoro-2-deoxy-D-glucose), the patient was assigned clinical stage IIBX (fig. 1), with a high age-adjusted international prognosis index.

In order to evaluate the functional status of the patient, a CGA was performed and brought up multiple comorbidities (long-lasting hypertension, chronic arterial vasculopathy, 
end-stage renal disease, depression and osteoarthrosis of the knee and elbows), polypharmacy, an increased risk for falls due to the osteoarthrosis and medications used, minimal dependency for activities of daily living (difficulty only in transferring) and significant dependency for instrumental activities of daily living, only being able to use the telephone without help, with a score of 11 . His performance status was graded as 2 , and we found a CIRS-G (Cumulative Illness Rating Scale - Geriatric) score of 9 and a severity index of 3 . The mini-nutritional assessment denoted a risk for malnutrition, with a body mass index of 23 and albumin of $4 \mathrm{~g} / \mathrm{dl}$ at diagnosis. Furthermore, the SOF (Study of Osteoporotic Fractures) frailty score [7] was positive - the patient was unable to rise from a chair 5 times without using the arms, and he reported a reduced energy level. In respect to psychosocial aspects, his social support and caregivers were his wife and son, and no distress factors were identified among them. Importantly, the patient's self-reported general health status was rated as 'good', in a scale with options between excellent, good, fair and poor.

In summary, information from the CGA and the results of the SOF index indicated that we were dealing with a frail patient. Nevertheless, we believed that, with geriatric intervention, nutritional monitoring and physiotherapy, the patient could tolerate a tailored treatment.

After proper evaluation of cardiac function, an adapted chemotherapy regimen was proposed which consisted of R-mini-CHOP [4] every 21 days, with the following doses: 375 $\mathrm{mg} / \mathrm{m}^{2}$ rituximab, $25 \mathrm{mg} / \mathrm{m}^{2}$ doxorubicin, $400 \mathrm{mg} / \mathrm{m}^{2}$ cyclophosphamide and $1 \mathrm{mg}$ vincristine on day 1 of each cycle and $40 \mathrm{mg} / \mathrm{m}^{2}$ oral prednisone on days $1-5$. Additionally, doses of doxorubicin and cyclophosphamide were reduced by 10\%, following directions of dose adjustment in elderly cancer patients with renal insufficiency. During the treatment, the patient went to regular dialysis sessions 3 times per week. Although no drugs in this chemotherapy scheme are significantly removed by the HD, an extra session was performed $12 \mathrm{~h}$ after each chemotherapy administration in order to improve drug tolerance [8]. Prophylactic granulocyte colony-stimulating factor was not given; hematologic toxicity was accessed every other week with complete blood cell counts. The patient had a good tolerance to the treatment, with no adverse events and/or grade 3/4 toxicities. No clinical deterioration was observed by the CGA during chemotherapy. After 6 cycles of R-mini-CHOP, the patient achieved unconfirmed complete remission (fig. 2) and consolidation radiotherapy was performed (36 Gy). Two months after completion of radiotherapy, the patient presented with mild congestive heart failure, functional class II. At the last follow-up, he was still in unconfirmed complete remission, with a progression-free survival of 11.3 months and no further deterioration of his previous functional status.

\section{Discussion}

Lymphoma in elderly patients is a common clinical scenario, although the optimal therapeutic approach to such patients has not been established. Moreover, the main challenge of this case was how to choose a suitable treatment for a frail older patient undergoing HD.

Foremost, as a guide to the decision-making process, the geriatric assessment played a central role in understanding our patient's functional reserve and social support. The CGA is a tool developed by geriatricians to evaluate the elderly exploring the functional status, emotional conditions, social support, nutritional status, polypharmacy and the presence of geriatric syndromes [9]. With the data provided by the CGA, oncologists possess relevant information about the physiological status of the patient, which helps to avoid subjective decisions of who should or should not be treated based only on chronological age. 
A major issue encountered by oncologists treating older cancer patients is how to select patients suitable for standard or attenuated therapy. Regarding this, the recognition of a frail person is of critical importance. Definition of frailty is controversial, and the most accepted description is the one proposed by Fried et al. [10], derived from the Cardiovascular Health Study (CHS), in which 3 or more of the following 5 components have to be present: unintentional weight loss, self-reported reduced energy level, reduced grip strength, slow walking speed and a low level of physical activity. The CHS index, however, is a complex and time-consuming method and other scales are often used to screen frailty. Thus, a practical clinical tool commonly used is the SOF index [7], in which frailty is identified by the presence of 2 or more of the following 3 items: weight loss, the subject's inability to rise from a chair 5 times without using the arms, and reduced energy level. Thus, our patient was at increased risk of toxicities, requiring an oncogeriatric management and tailored treatment plan.

Referring to the burden of comorbidities on treatment selection, another capital concern about this case was related to the patient's chronic renal failure, as few data is available about the management of such patients undergoing chemotherapy. Pharmacokinetic studies on rituximab in patients with end-stage renal disease demonstrated that this agent is not eliminated by dialysis [11]. Similarly, the use of vincristine is safe in severe renal failure $(<30$ $\mathrm{ml} / \mathrm{min}$ ), with no need for dose adjustment [8]. On the other hand, controversial data exists regarding doxorubicin. In theory, doxorubicin's main active metabolite (doxorubicinol) is not predominantly eliminated by the urinary tract $( \pm 10 \%)$ [12], not requiring dose adjustment. Currently, there are no guidelines for dose adjustment of doxorubicin in renal impairment, but Janus et al. [8] suggest that the drug should be administered on the day without HD or after it. As regards cyclophosphamide, between 30 and $60 \%$ of its dose is eliminated by the kidneys as the drug itself or as its metabolites and, consequently, its pharmacokinetics can be modified in renal insufficiency patients [13]. Hence, it is suggested to reduce the dose of cyclophosphamide by $25 \%$ in HD patients [8] and to deliver the drug after HD sessions. Janus et al. [8] also suggest the use of partial dialysis removal to improve drug tolerance, recommending starting HD sessions at a certain time following chemotherapy to remove the drugs that have not been distributed to the site of action and could generate side effects. Based on this rationale, we judged it reasonable to submit our patient to an extra HD session $12 \mathrm{~h}$ after each chemotherapy cycle. Indeed, the patient experienced no hematological toxicity related to the treatment.

Given the previously exposed clinical scenario about this patient's functional status and comorbidities, he could have been considered only for best supportive care or palliative chemotherapy. Nonetheless, as we were the first to present a case of DLBCL, a malignancy with a high chance of response to treatment and cure, and supported by the geriatric intervention, we decided to start treatment with curative intent. Indeed, lymphoma in elderly patients is not less responsive to treatment than in younger patients, and the major issue is to choose the adequate chemotherapy and to achieve a long-lasting complete response. In a randomized trial by the GELA group in patients aged 60-80 years, the combination of CHOP and rituximab was significantly superior to CHOP alone in terms of complete response and survival [14]. In 2011, the same group [4] studied the efficacy of a low-dose CHOP regimen and rituximab (R-mini-CHOP), in a prospective study with patients older than 80 years with DLBCL, achieving a 2-year overall survival of $59 \%$ and a 2-year progression-free survival of $47 \%$, with $62 \%$ of the patients achieving a complete or unconfirmed complete response rate. The great balance between efficacy and safety of this attenuated immunochemotherapy guided the decision to choose R-mini-CHOP to treat our patient; in addition, we implemented a reduction of $10 \%$ in doxorubicin and cyclophosphamide doses. 
Probably, the choice of an anthracycline-containing regimen was essential to achieve a good outcome in our patient, as shown in other series $[3,5,6]$. However, a great concern refers to the cardiotoxicity related to anthracyclines. In the study of Peyrade et al. [4], Rmini-CHOP in elderly patients was associated with a $6 \%$ rate of serious cardiac adverse events. Additional data shows that the risk of developing congestive heart failure after 8 years for an elderly patient receiving an anthracycline dose of $25-35 \mathrm{mg} / \mathrm{m}^{2}$ per cycle, repeated for 6 cycles, is only marginal [9]. Nevertheless, our patient developed congestive heart failure, functional class II, although we cannot warrant that it was strictly related to doxorubicin, given other concomitant risk factors were also present (long-lasting hypertension, chronic arterial vasculopathy, end-stage renal disease and radiotherapy). For patients with known altered cardiac function, alternative regimens such as COP (cyclophosphamide, vincristine and prednisone) are reasonable, although their response rates are low. For example, in a prospective study conducted by Soubeyran et al. [15], COP was associated with only $18.5 \%$ of complete responses. Indeed, when planning the chemotherapy regimen, it is essential to question if there really is any contraindication to the use of CHOP-based regimens, as patients not treated with CHOP have a worse survival and a higher death rate from lymphoma [9].

In conclusion, R-mini-CHOP represented a reasonable treatment option for this senior adult with renal failure, with good disease control and no relevant hematological toxicity. The information from the CGA and an oncogeriatric approach led to a successful management of this frail patient, highlighting that an adapted plan of care is a key issue to improve outcomes of elderly cancer patients.

\section{References}

1 Jemal A, Siegel R, Ward E, et al: Cancer statistics 2007. CA Cancer J Clin 2007;57:43-66.

-2 Morton LM, Wang SS, Devesa SS, Hartge P, Weisenburger DD, Linet MS: Lymphoma incidence patterns by WHO subtype in the United States, 1992-2001. Blood 2006;107:265-276.

-3 Bastion Y, Blay JY, Divine M, et al: Elderly patients with aggressive non-Hodgkin's lymphoma: disease presentation, response to treatment and survival - a Groupe d'Etude des Lymphomas de l'Adulte study on 453 patients older than 69 years. J Clin Oncol 1997;15:2945-2953.

-4 Peyrade F, Jardin F, Thieblemont C, Thyss A: Attenuated immunochemotherapy regimen (R-miniCHOP) in elderly patients older than 80 years with diffuse large B-cell lymphoma: a multicentre, single-arm, phase 2 trial. Lancet Oncol 2011;12:460-468.

5 Bairey 0, Benjamini O, Blickstein D, Elis A, Ruchlemer R: Non-Hodgkin's lymphoma in patients 80 years of age or older. Ann Oncol 2006;17:928-934.

6 Thieblemont C, Grossoeuvre A, Houot R, et al: Non-Hodgkin's lymphoma in very elderly patients over 80 years. A descriptive analysis of clinical presentation and outcome. Ann Oncol 2008;19:774-779.

7 Ensrud KE, Ewing SK, Taylor BC, et al: Comparison of 2 frailty indexes for prediction of falls, disability, fractures and death in older women. Arch Intern Med 2008;168:382-389.

-8 Janus N, Thariat J, Boulanger H, Deray G, Launay-Vacher V: Proposal for dosage adjustment and timing of chemotherapy in hemodialyzed patients. Ann Oncol 2010;21:1395-1403.

-9 Peyrade F, Gastaud L, Ré D, Pacquelet-Cheli S, Thyss A: Treatment decisions for elderly patients with haematological malignancies: a dilemma. Lancet Oncol 2012;13:e344-e352.

-10 Fried LP, Tangen CM, Walston J, et al: Frailty in older adults: evidence for a phenotype. J Gerontol A Biol Sci Med Sci 2001;56:M146-M156.

11 Ostronoff F, Ostronoff M, Florencio R, et al: Safety of fractionated dose of rituximab in renal failure patients receiving hemodialysis. Leuk Lymphoma 2006;47:757-759. 


\section{Case Reports in Oncology}

\begin{tabular}{l|l}
\hline Case Rep Oncol 2013;6:197-203 \\
\hline DOI: 10.1159/000350746 & $\begin{array}{l}\text { ○ 2013 S. Karger AG, Basel } \\
\text { www.karger.com/cro }\end{array}$ \\
\hline
\end{tabular}

Pontes et al.: Treatment of a Frail Older Patient with Diffuse Large B-Cell Lymphoma on Maintenance Dialysis: Attenuated Immunochemotherapy and Adapted Care Plan

12 Gabizon A, Catane R, Uziely B, et al: Prolonged circulation time and enhanced accumulation in malignant exudates of doxorubicin encapsulated in polyethylene-glycol coated liposomes. Cancer Res 1994;54:987992.

11 De Jonge ME, Huitema AD, Rodenhuis S, et al: Clinical pharmacokinetics of cyclophosphamide. Clin Pharmacokinet 2005;44:1135-1164.

14 Coiffier B, Lepage E, Briere J, et al: CHOP chemotherapy plus rituximab compared with CHOP alone in elderly patients with diffuse large-B-cell lymphoma. N Engl J Med 2002;346:235-242.

15 Soubeyran P, Khaled H, MacKenzie M, et al: Diffuse large B-cell and peripheral T-cell non-Hodgkin's lymphoma in the frail elderly. A phase III EORTC trial with a progressive and cautious treatment emphasizing geriatric assessment. J Geriatr Oncol 2011;2:36-44.
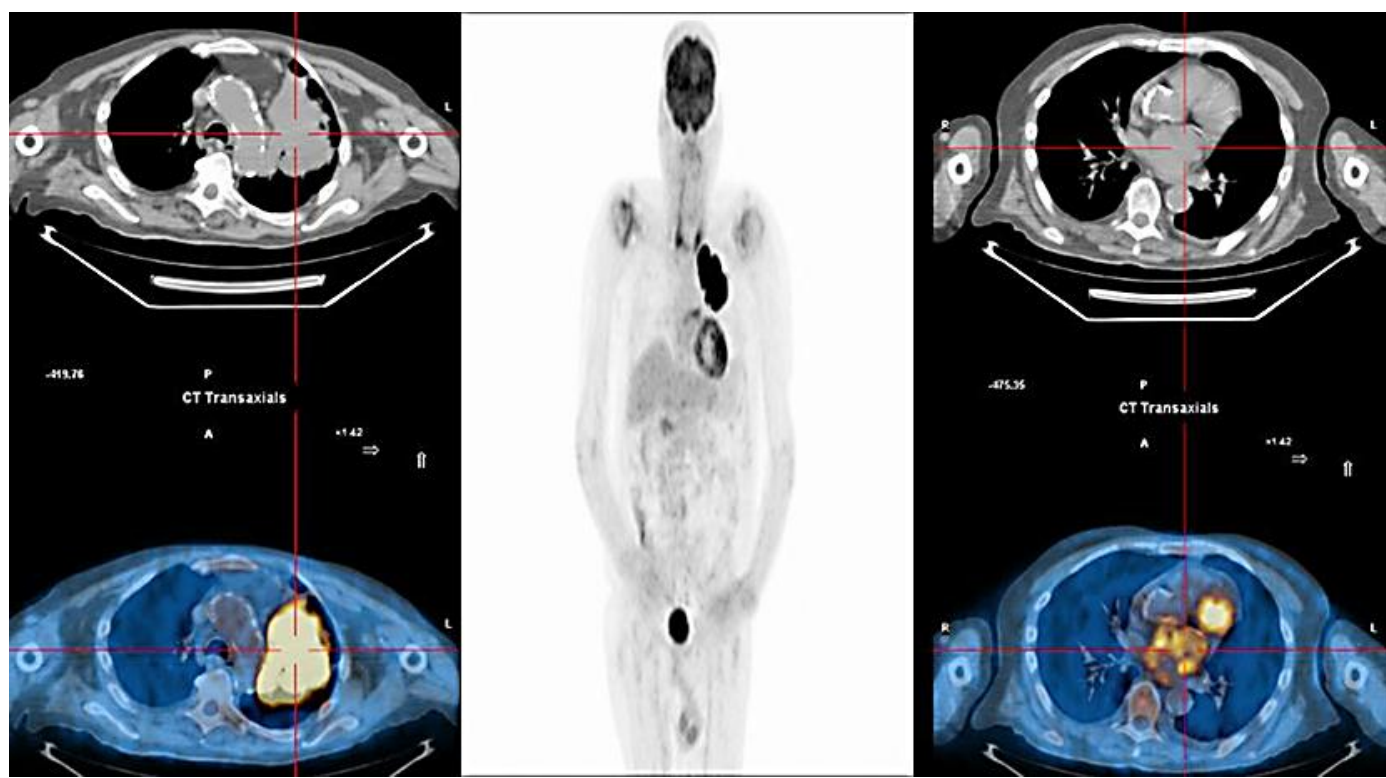

Fig. 1. PET/CT at diagnosis showing an $8.3 \times 9.8 \mathrm{~cm}^{2}$ mass on the left upper lobe of the lung (SUV 35) and mediastinal lymph nodes (SUV 4.3). 


\section{Case Reports in Oncology}

\begin{tabular}{l|l}
\hline Case Rep Oncol 2013;6:197-203 \\
\hline DOI: $10.1159 / 000350746$ & $\begin{array}{l}\text { C 2013 S. Karger AG, Basel } \\
\text { www.karger.com/cro }\end{array}$ \\
\hline
\end{tabular}

Pontes et al.: Treatment of a Frail Older Patient with Diffuse Large B-Cell Lymphoma

on Maintenance Dialysis: Attenuated Immunochemotherapy and Adapted Care Plan

\section{Prior to treatment}

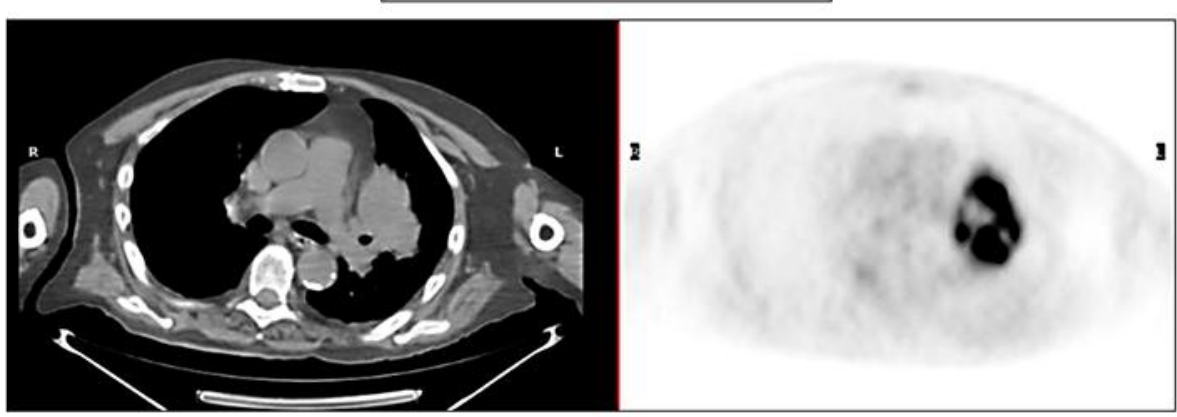

After 6 cycles of R-mini-CHOP

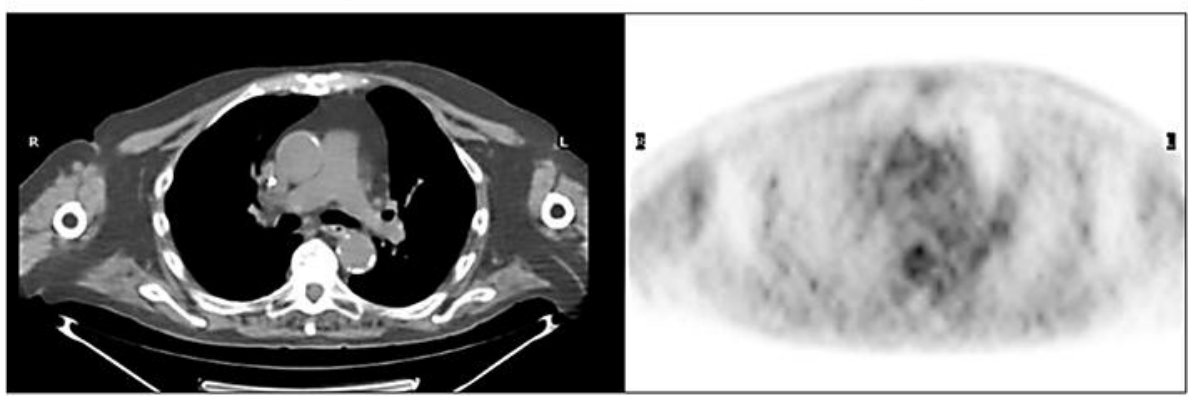

Fig. 2. PET/CT after 6 cycles of R-mini-CHOP showing a complete response. 